\title{
Patterns of pneumococcal vaccination and revaccination in elderly and non-elderly adults: a Vaccine Safety Datalink study Lisa A Jackson*1, Roger Baxter ${ }^{2}$, Allison L Naleway ${ }^{3}$, Edward A Belongia ${ }^{4}$ and James Baggs ${ }^{5}$
}

Address: ${ }^{1}$ Group Health Center for Health Studies, Group Health Cooperative, Seattle, USA, ${ }^{2}$ Kaiser Permanente Vaccine Study Center, Kaiser Permanente of Northern California, Oakland, USA, ${ }^{3}$ Center for Health Research, Kaiser Permanente Northwest, Portland, USA, ${ }^{4}$ Epidemiology Research Center, Marshfield Clinic Research Foundation, Marshfield, USA and ${ }^{5}$ Immunization Safety Office, Centers for Disease Control and Prevention, Atlanta, USA

Email: Lisa A Jackson* - jackson.l@ghc.org; Roger Baxter - Roger.baxter@kp.org; Allison L Naleway - Allison.naleway@kpchr.org; Edward A Belongia - Belongia.edward@marshfieldclinic.org; James Baggs - jbaggs@cdc.gov

* Corresponding author

Published: 25 March 2009

BMC Infectious Diseases 2009, 9:37 doi:10.1/86/147/-2334-9-37
Received: 2 October 2008

Accepted: 25 March 2009

This article is available from: http://www.biomedcentral.com/147I-2334/9/37

(C) 2009 Jackson et al; licensee BioMed Central Ltd.

This is an Open Access article distributed under the terms of the Creative Commons Attribution License (http://creativecommons.org/licenses/by/2.0), which permits unrestricted use, distribution, and reproduction in any medium, provided the original work is properly cited.

\begin{abstract}
Background: Pneumococcal polysaccharide vaccine (PPV) is recommended for all adults 65 years of age and older and for younger adults with high-risk conditions. While data from national surveys provide information on the proportion of adults 65 years of age and older reporting ever receipt of PPV they do not collect more detailed information, such as age at vaccination or the total number of vaccinations received. In addition, there is relatively little information available on PPV coverage in younger adults with chronic conditions. To assess contemporary patterns of pneumococcal vaccination and revaccination of adults, we conducted a cross-sectional study of adults enrolled in medical care organizations (MCOs) participating in the Vaccine Safety Datalink project.
\end{abstract}

Methods: The study population included 1.5 million adults 25 years of age and older enrolled in the four participating MCOs on December I, 2006. PPVs administered to members of the study population prior to that date were identified from computerized immunization registries maintained by the MCOs.

Results: Among the general population of adults 25 through 64 years of age, vaccine coverage increased from $2 \%$ in the $25-29$ year old age-group to $26 \%$ in the $60-64$ year old age-group. In all age-groups, coverage was substantially higher in persons defined as having a chronic high risk condition. This was particularly true for diabetes mellitus, with vaccine coverage of over $50 \%$ in the lower age-groups and 75\% in those 60-64 years of age. Among adults 65 years of age and older, $82 \%$ had received at least one PPV and $18 \%$ had received two or more PPVs.

Conclusion: We found higher levels of PPV coverage among adults 65 years of age and older and among younger adults with diabetes mellitus than reported by national surveys and for those groups PPV coverage approached the Healthy People 2010 national objectives. These results suggest that achieving those objectives for PPV is possible and that high vaccination coverage may be facilitated by vaccine tracking and reminder systems. 


\section{Background}

In the United States, pneumococcal polysaccharide vaccine (PPV) is recommended for all adults 65 years of age and older and for younger adults with certain chronic conditions, such as diabetes mellitus [1]. Seniors who received one or more doses of PPV when younger than 65 should receive another dose on or after their $65^{\text {th }}$ birthday (and at least five years after the prior vaccination). Once vaccinated after the age of 65, however, no additional doses of PPV are recommended, and revaccination is not recommended for immunocompetent persons under age 65. National surveys such as the Behavioral Risk Factor Surveillance System (BRFSS) survey indicate that pneumococcal vaccine coverage in adults $\geq 65$ years has greatly increased over the past two decades, with $66 \%$ of adults $\geq 65$ years interviewed in the 2005 BRFSS survey reporting past receipt of PPV [2].

While data from the BRFSS and similar surveys provide information on the proportion of seniors reporting ever receipt of PPV they do not collect more detailed information, such as age at vaccination or the total number of vaccinations received. In addition, there is relatively little information available on PPV coverage in younger adults with chronic conditions.

Additional information on current patterns of PPV use in adults $\geq 65$ years and younger adults would be helpful in decision making regarding current and future strategies for prevention of pneumococcal infection. For example, knowledge of the proportion of adults $\geq 65$ years who received PPV before age 65 would aid in decisions regarding possible strategies for use of newer pneumococcal vaccines, for which the immunologic response may differ in PPV naïve and previously vaccinated persons. To assess contemporary patterns of pneumococcal vaccination and revaccination of adults, we conducted a cross-sectional study of approximately 1.5 million adults enrolled in medical care organizations (MCOs) participating in the Vaccine Safety Datalink project.

\section{Methods}

The Vaccine Safety Datalink project was established in 1991 to monitor and evaluate vaccine safety and includes eight participating MCOs with a combined patient population of over 8.8 million members. Project staff at each MCO prepare annual data files that contain demographic and medical information derived from MCO administrative data systems. These data files include information on age, sex, health plan enrollment, vaccination, and ambulatory and inpatient medical encounters. The specific goals, structure, and data characteristics of the Vaccine Safety Datalink have been described previously [3,4].
The study population for this assessment included adults 25 years of age and older who were enrolled in a participating MCO on December 1, 2006 and who had been continuously enrolled for at least five years prior to that date. Participating MCOs included Northern California Kaiser Permanente, Northwest Kaiser Permanente (Oregon), Group Health Cooperative (Washington), and Marshfield Clinic (Wisconsin). These MCOs were selected for this study from among the eight MCOs in the Vaccine Safety Datalink because of the availability of historical data on adult vaccinations.

Pneumococcal polysaccharide vaccinations administered to members of the study population prior to December 1 , 2006 were identified from computerized immunization registries maintained by the MCOs. For persons with more than one PPV recorded, the earliest vaccination recorded was defined as the first vaccination and subsequent vaccinations were defined as separate vaccinations if there was an interval of at least 91 days since the prior vaccination. If the interval between two consecutive recorded vaccinations was 90 days or less the more recent vaccination was defined as a duplicate and was not counted as a separate vaccination. This interval was selected because repeated pneumococcal vaccinations should not be administered within a 90 day interval and data entry errors can occur. Multiple entries within this window were rare and a second PPV with an administration date less than 91 days following a prior PPV accounted for only 1949 of the 563,524 (0.3\%) PPVs identified.

The presence or absence of chronic conditions was defined by International Classification of Diseases $9^{\text {th }}$ Revision Clinical Modification (ICD9-CM) codes assigned to inpatient or outpatient medical encounters during 2006. Diabetes mellitus was defined by ICD9-CM code 250, congestive heart failure (CHF) by codes 402.01, 402.11, 402.91, 404.01, 404.03, 404.11, 404.13, 404.91, 404.93, 425 , and 428 , and chronic obstructive pulmonary disease (COPD) by codes 491.1 through 492.8, 494, and 496. These conditions were selected for evaluation of vaccination patterns because they are generally not transient and expected to be reasonably common in adults under 65 years of age. In addition, a category of no disease was defined by the absence of the codes listed above as well as codes for ischemic cardiac disease (410 through 414), asthma (493), emphysema (510), cancer (140-155, 157$171,173-208)$, renal disease (580-588, 591, V56), rheumatologic disease $(710,714)$, and organ transplantation (996).

The study was approved by the Institutional Review Boards of the participating MCOs. 


\section{Results}

The study population included $1,527,357$ adults 25 years of age and older. Of those, 421,677 (28\%) were 65 years of age and older and 831,869 (54\%) were female. The proportion of adults with at least one PPV recorded prior to December 1, 2006, by year of age, is shown in Figure 1.

\section{Adults 25 through 64 years of age}

Among the general population of adults 25 through 64 years of age, vaccine coverage increased from $2 \%$ in the 25-29 year old age-group to $26 \%$ in the 60-64 year old age-group (Table 1 ). In all age-groups, coverage was substantially higher in persons defined as having a chronic high risk condition. This was particularly true for diabetes mellitus, with vaccine coverage of over $50 \%$ in the lower age-groups and 75\% in those 60-64 years of age.

Revaccination was generally uncommon in adults 25 through 64 years of age, although there were trends for a higher prevalence of revaccination in persons with chronic disease and in the older age-groups. The highest prevalence of revaccination, $17 \%$, was in persons $60-64$ years of age with $\mathrm{CHF}$.

\section{Adults $\mathbf{6 5}$ years of age and older}

Eighty-two percent of adults 65 years of age and older had ever received PPV and $73 \%$ had received at least one PPV on or after age 65 (Table 2). There were no differences in patterns of receipt of PPV by sex; vaccine coverage was $82 \%$ in females and $81 \%$ in males. Vaccine coverage for each of the four MCOs was $74 \%, 81 \%, 86 \%$, and $87 \%$; differences in coverage were not due to differences in age distribution of the populations in the MCOs. Overall, lower coverage (67\%) was seen in the 65-69 year old agegroup than in the older age-groups and coverage among those 70 and older was $87 \%$. Persons with chronic illness tended to have higher vaccination coverage.

Eighteen percent of adults $\geq 65$ years had two or more PPVs recorded and 7\% had received at least two PPVs on or after age 65. Nearly all who received more than one PPV had a total of two vaccinations recorded; only $0.6 \%$ had three or more PPVs recorded. Among those who received two PPVs on or after age 65 , the interval between the vaccinations was less than five years for $25 \%$, was from five through seven years in $43 \%$, and was eight years or more for the remaining $32 \%$.

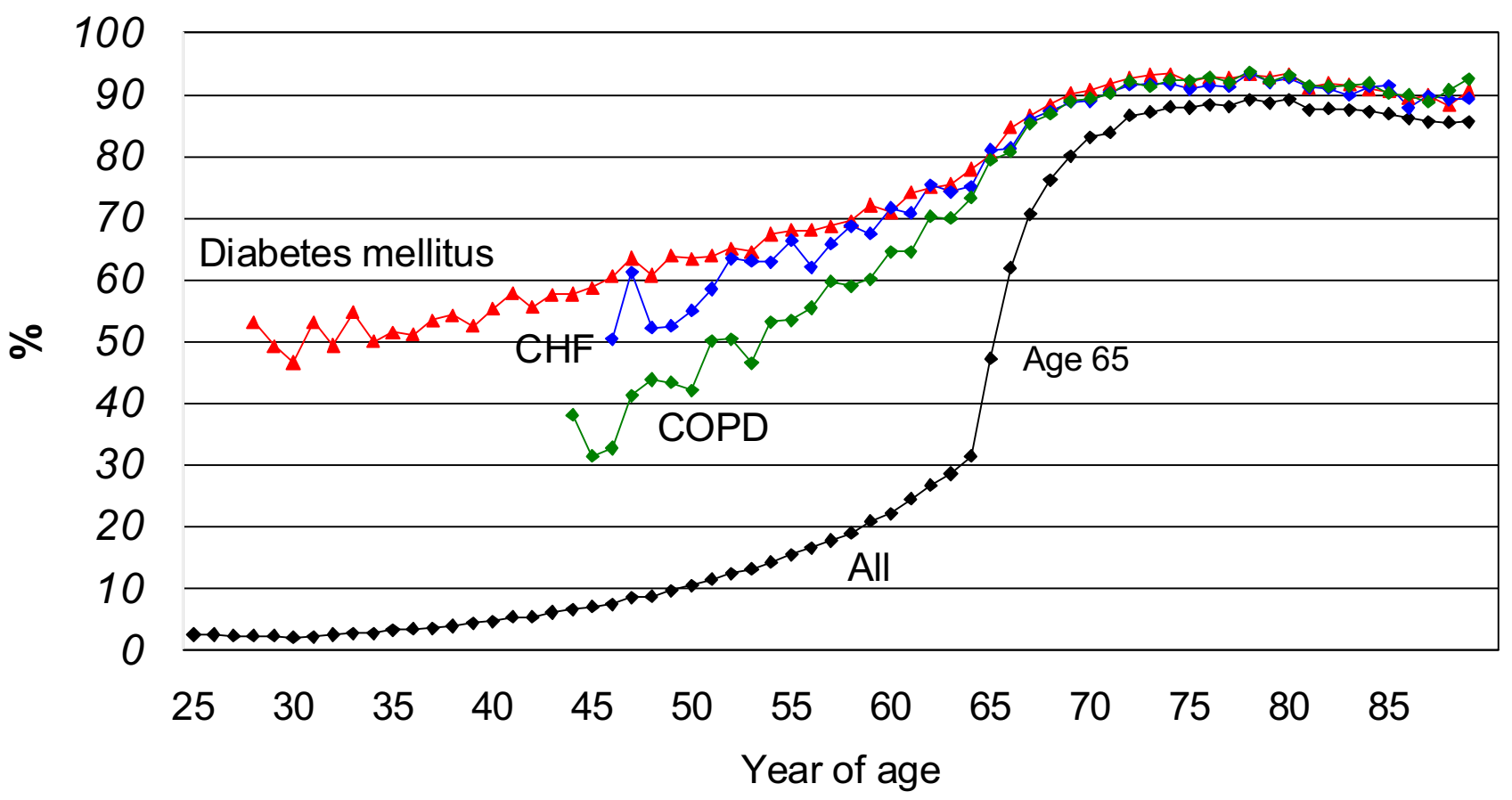

Figure I

Proportion of adults 25-89 years of age with ever receipt of PPV, by year of age and chronic condition. Values are shown only for year of age categories with a denominator of at least 100 persons. The age groups shown include 28-89 years for diabetes mellitus, 46-89 years for CHF, 44-89 years for COPD, and 25-89 for all. CHF, congestive heart failure. COPD, chronic obstructive pulmonary disease. 


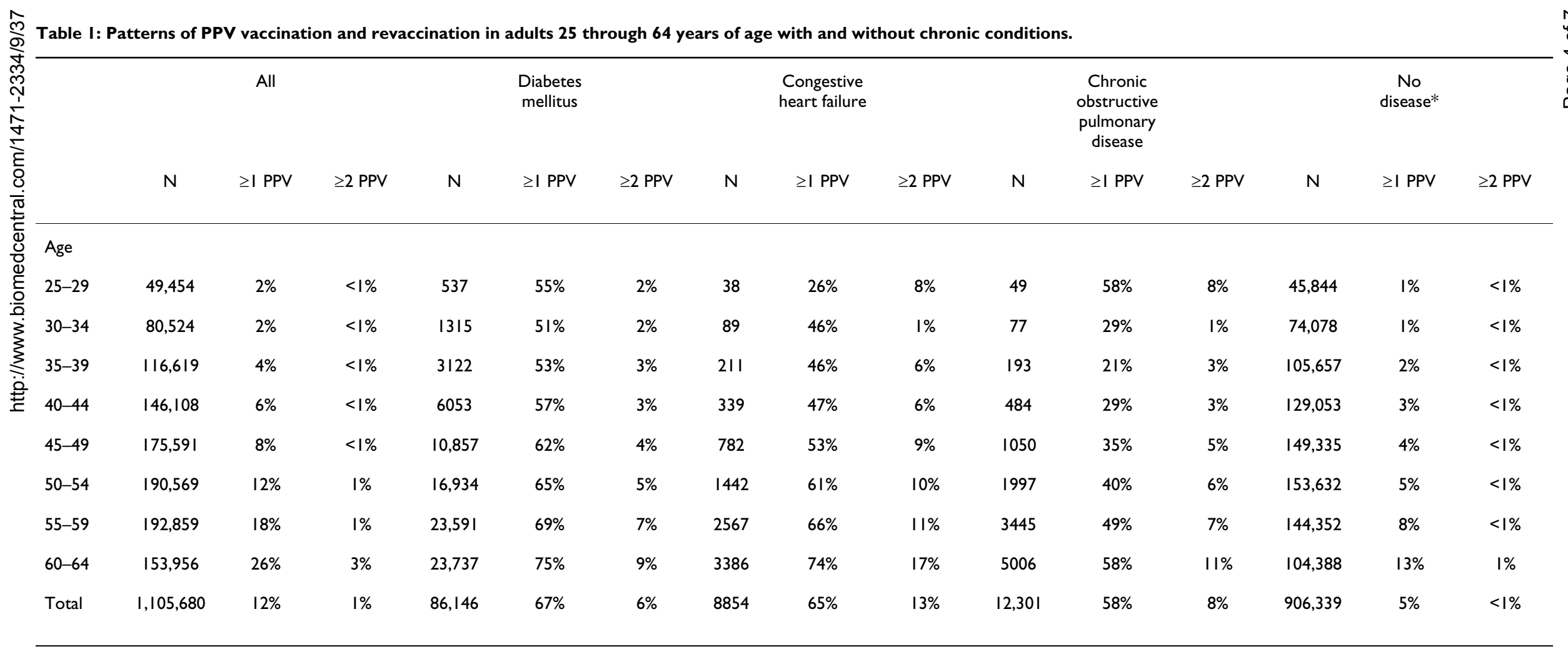

*See methods for the definition of persons with no disease. 
Table 2: Patterns of PPV vaccination and revaccination in adults $\geq 65$ years.

\begin{tabular}{|c|c|c|c|c|c|c|}
\hline & $\mathrm{N}$ & $\geq$ I PPV at any age & $\geq$ I PPV prior to age 65 & $\geq 1 \mathrm{PPV}$ on or after age 65 & $\geq 2$ PPV ever & $\geq 2$ PPV on or after age 65 \\
\hline Age & & & $\%$ & & & \\
\hline $65-69$ & 111,019 & 67 & 34 & 44 & 15 & $<1$ \\
\hline 70-74 & 105,789 & 86 & 28 & 78 & 23 & 3 \\
\hline 75-79 & 87,328 & 88 & 10 & 87 & 18 & 11 \\
\hline $80-84$ & 65,758 & 88 & 1 & 88 & 14 & 14 \\
\hline $85-89$ & 36,033 & 86 & $<1$ & 86 & 14 & 14 \\
\hline $90+$ & 15,750 & 83 & 0 & 83 & 13 & 13 \\
\hline$\geq 65$ & 421,677 & 82 & 18 & 73 & 18 & 7 \\
\hline$\geq 65$ with diabetes & 86,258 & 91 & 31 & 77 & 27 & 8 \\
\hline$\geq 65$ with CHF & 35,328 & 90 & 17 & 83 & 24 & 12 \\
\hline$\geq 65$ with COPD & 36,623 & 90 & 23 & 82 & 27 & 12 \\
\hline
\end{tabular}

CHF, congestive heart failure. COPD, chronic obstructive pulmonary disease.

The patterns of age at the time of first pneumococcal vaccination among adults $\geq 65$ years demonstrate changes in PPV availability and use over time (Table 3 ). Adults in their 80s tended to have received their first PPV in their 70 s or later, while younger persons had a greater likelihood of having received PPV before age 65 or soon after age 65 .

\section{Discussion and Conclusion}

In this assessment of 1.5 million adults enrolled in four MCOs we found much higher levels of PPV coverage than has been reported in national surveys of the United States population. For example, $82 \%$ of adults $\geq 65$ years in our study population had received at least one PPV, compared with a median state specific coverage rate among adults $\geq 65$ years of $66 \%$ in the 2005 BRFSS survey and 57\% in the 2006 National Health Interview Survey (NHIS) [2]. We also found much higher rates of coverage among adults less than 65 years of age with diabetes mellitus than has been reported by those national surveys. In our study population, over half of adults less than 65 years of age with diabetes had received PPV, even in the youngest age groups, compared to the lower estimates of coverage in these age groups reported by the 2005 BRFSS survey (Table 4).

Possible reasons for the higher vaccination coverage that we observed include our assessment of vaccination status based on data from computerized immunization registries, which may be a more sensitive indicator of past vaccination than self-report [3]. In addition, our MCO study population included, by definition, only insured persons, a group that is likely have better access to preventive care services than uninsured persons. Further, the MCOs included in this study were selected on the basis of availability of historical adult vaccination data, which may also indicate a greater focus on immunization services in those organizations. During the study period some of the participating MCOs had systems to prompt providers to administer PPV to an eligible adult which likely facilitated vaccine delivery. Lastly, we defined diabetes mellitus and other chronic conditions by diagnostic codes assigned to medical encounters, and so all persons defined as having a chronic condition in our analyses had at least one med-

Table 3: Age at receipt of first recorded PPV, for adults $\geq 65$ years with at least one prior PPV recorded.

\begin{tabular}{|c|c|c|c|c|c|c|}
\hline & \multicolumn{5}{|c|}{ Age as of December I, 2006} & \multirow[b]{2}{*}{$90+$} \\
\hline & $65-69$ & $70-74$ & $75-79$ & $80-84$ & $85-89$ & \\
\hline Age at first recorded PPV & & & $\mathrm{N}(\%)$ & & & \\
\hline $40-44$ & $3(<1)$ & 0 & 0 & 0 & 0 & 0 \\
\hline $45-49$ & $60(<1)$ & $6(<1)$ & 0 & 0 & 0 & 0 \\
\hline $50-54$ & $1824(2)$ & $114(<1)$ & $28(<1)$ & 0 & 0 & 0 \\
\hline $55-59$ & $12,612(17)$ & $3296(4)$ & $216(<1)$ & $26(<1)$ & 0 & 0 \\
\hline $60-64$ & $23,698(32)$ & $25,990(29)$ & $8129(10)$ & $492(<1)$ & $56(<1)$ & 0 \\
\hline $65-69$ & $35,957(48)$ & $53,893(59)$ & $38,933(50)$ & $10,613(18)$ & $696(2)$ & $34(<1)$ \\
\hline $70-74$ & - & $7321(8)$ & $24,717(32)$ & $24,530(42)$ & $6194(20)$ & $330(2)$ \\
\hline $75-79$ & - & - & $5194(7)$ & $18,382(32)$ & $\mid 2,652(4 \mid)$ & $2278(17)$ \\
\hline $80-84$ & - & - & - & $376 \mid(6)$ & $9563(31)$ & 4790 (37) \\
\hline $85-89$ & - & - & - & - & $1859(6)$ & $4034(31)$ \\
\hline $90+$ & - & - & - & - & - & 1577 (I2) \\
\hline Total & 74,154 & 90,620 & 77,217 & 57,804 & 31,020 & 13,043 \\
\hline
\end{tabular}


Table 4: Estimates of pneumococcal polysaccharide vaccine coverage in the United States from the 2005 Behavioral Risk Factor Surveillance System survey.

\begin{tabular}{|c|c|c|c|}
\hline Group & Age & $\%$ ever had pneumonia shot & \\
\hline & & All & Diabetes* \\
\hline \multirow[t]{9}{*}{ All } & $25-29$ & 10 & 29 \\
\hline & $30-34$ & 9 & 17 \\
\hline & $35-39$ & 10 & 27 \\
\hline & $40-44$ & 10 & 30 \\
\hline & $45-49$ & 11 & 30 \\
\hline & $50-54$ & 14 & 34 \\
\hline & $55-59$ & 21 & 41 \\
\hline & $60-64$ & 30 & 48 \\
\hline & $65+$ & 64 & 69 \\
\hline \multirow[t]{2}{*}{ White, Non-Hispanic } & $18-64$ & 14 & 40 \\
\hline & $65+$ & 67 & 75 \\
\hline \multirow[t]{2}{*}{ Black, Non-Hispanic } & $18-64$ & 16 & 33 \\
\hline & $65+$ & 50 & 55 \\
\hline \multirow[t]{2}{*}{ Hispanic } & $18-64$ & 13 & 23 \\
\hline & $65+$ & 42 & 47 \\
\hline \multirow[t]{2}{*}{ Has health care coverage } & $18-64$ & 14 & 38 \\
\hline & $65+$ & 64 & 70 \\
\hline \multirow[t]{2}{*}{ Does not have health care coverage } & $18-64$ & 13 & 27 \\
\hline & $65+$ & 42 & 52 \\
\hline
\end{tabular}

Data from the WEAT: Web Enabled Analysis Tool available at http://apps.nccd.cdc.gov/s broker/htmsql.exe/weat/Freq analysis.hsql.

* Ever told have diabetes (excluding pregnancy)

ical encounter in the prior year, and this may select for subgroups with higher vaccination coverage.

Data on race and ethnicity is generally not available in the MCO data systems and so we could not evaluate differences in vaccination coverage by those factors. Data from national surveys indicates that PPV coverage varies by race and ethnicity and is higher in white, non-hispanics than in either Hispanics or blacks (Table 4). If white persons were relatively overrepresented in our MCO populations then this could account in part for the variations in vaccine coverage estimates between our estimates and national estimates. The prevalence of pneumococcal vaccination in our population remained higher than national estimates for the white non-hispanic subgroup, however, suggesting that vaccination coverage was higher in the study population even after accounting for possible demographic differences.

Despite the lack of a recommendation for administration of a second PPV after age 65, receipt of two or more PPVs after age 65 was not uncommon. This may be due in part to a lack of understanding of the recommendations by some care providers. It is possible that the proportion of adults $\geq 65$ years who have received two or more PPVs will further increase as the prevalence of first vaccination in adults rises over time. Among adults 50-64 years of age with diabetes, CHF, or COPD, between 5 and $17 \%$ had received two PPVs, depending on the condition and age group.

The Healthy People 2010 objectives for pneumococcal vaccination in adults are to achieve $90 \%$ coverage of both institutionalized and non-institutionalized persons 65 years of age and older and to achieve $60 \%$ coverage of noninstitutionalized high-risk adults 18 through 64 years of age http://www.healthypeople.gov/document/html/ objectives/14-29.htm. As of 2006, PPV coverage in our study population approached the target for persons 65 years of age and older and for younger adults with diabetes or CHF. While there are differences between our study population of MCO members and the general U.S. population, these results suggest that achieving the Healthy People 2010 national objectives for pneumococcal vaccine is possible. Higher coverage may be facilitated by vaccine tracking and reminder systems. 


\section{Competing interests}

LAJ has served as a consultant to Merck, a manufacturer of pneumococcal polysaccharide vaccine. $\mathrm{RB}$ has received research funding from Merck. ALN, EAB, and JB declare that they have no competing interests.

\section{Authors' contributions}

LAJ developed the study concept and protocol and all other authors (RB, ALN, EAB, JB) reviewed the protocol and made contributions to study design. All authors participated in the acquisition of data and analysis and interpretation of the data. LAJ drafted the manuscript and all other authors were involved in revising it critically for important intellectual content and have given final approval of the version submitted.

\section{Acknowledgements}

We thank the Vaccine Safety Datalink staff members for their valuable data management, programming, and project management contributions, which enabled the assembly of the Vaccine Safety Datalink files. We also acknowledge the support of the staff at America's Health Insurance Plans.

This study was supported by the Vaccine Safety Datalink contract with America's Health Insurance Plans, funded by the Centers for Disease Control and Prevention (200-2002-00732).

\section{References}

I. CDC: Prevention of pneumococcal disease: recommendations of the Advisory Committee on Immunization Practices (ACIP). MMWR Recomm Rep 1997, 46:I-24.

2. CDC: Influenza and pneumococcal vaccination coverage among persons aged $>$ or $=65$ years - United States, 20042005. MMWR Morb Mortal Wkly Rep 2006, 55: 1065-1068.

3. Chen RT, Glasser JW, Rhodes PH, Davis RL, Barlow WE, Thompson RS, Mullooly JP, Black SB, Shinefield HR, Vadheim CM, Marcy SM, Ward JI, Wise RP, Wassilak SG, Hadler SC: Vaccine Safety Datalink project: a new tool for improving vaccine safety monitoring in the United States. The Vaccine Safety Datalink Team. Pediatrics 1997, 99:765-773.

4. DeStefano F: The Vaccine Safety Datalink project. Pharmacoepidemiol Drug Saf 2001, 10:403-406.

\section{Pre-publication history}

The pre-publication history for this paper can be accessed here:

http://www.biomedcentral.com/1471-2334/9/37/prepub http.//www.biomedcentral.com/1471-2334/9/37 